\title{
Laser ablation at the hydrodynamic regime
}

\author{
Ardian B. Gojani ${ }^{1, a}$ \\ ${ }^{1}$ Institute of Fluid Science, Tohoku University, 2-1-1 Katahira, Aoba, 980-8577 Sendai, Japan
}

\begin{abstract}
Laser ablation of several metals and PVC polymer by high energy nanosecond laser pulses is investigated experimentaly. Visualization by shadowgraphy revealed the dynamics of the discontinuities in ambient air and ablation plume above the target surface, while surface profiling allowed for determination of the ablated mass.
\end{abstract}

\section{Introduction}

Laser ablation is the process by which a relatively small amount of material is removed from material's surface by means of a laser beam. The interaction between the laser beam and the material depends on three major systems:

- the laser beam, characterized by laser energy, pulse duration, wavelength, polarization, etc.

- the beam transmission system, which influences the spot size of the focused beam, determines the angle of incidence, and also accounts for the ambient surrounding the target, as well as the state of confinement, and

- the target material, which is characterized by its chemical composition and microstructure, expressed through a variety of parameters such as reflectance and absorption coefficient, thermal diffusivity, potentials of ionization, and others.

The wide ranges of values that these parameters can have cause very different ablation mechanisms, which also can be utilized for a variety of purposes, as it is discussed in detail in the textbook of Bäuerle, [1].

The motivation for the present work stems from the potential of using laser ablation for propelling the ablated foil, or for accelerating microparticles deposited at the rear end of the ablated foil, such that the microparticles can be ballistically delivered. Previous works that describe these applications can be found in references [2-4]. The aim of this work is to outline our understanding of ablation mechanisms and find the range of values for the most influential parameters, such that optimized laser propulsion is achieved. For this reason, an experimental study of laser ablation by high energy nanosecond laser pulses of several metals (aluminum, copper, tin, titanium, zinc, as well as stainless steel as a metallic material) and a polymer (hardened black polyvinyl chloride - PVC) is conducted.

Light falling on the surface of a condensed matter will excite the structure of the material, be it through free electrons in metals, or through intensification of vibrations of molecules in a polymer. The excitation energy, which at the initial stages is not thermal, will be dissipated into heat through collisions and electron-phonon coupling in a very short time. Typical relaxation times for metals are shorter than a few picoseconds, while for polymers can be as long

\footnotetext{
a e-mail: gojani@edge.ifs.tohoku.ac.jp
}

as a few microseconds. If the phenomena in consideration are longer than the relaxation times, - as is the case in this study, - the laser can be considered as a heat source. Material response to increase of irradiance can be summarized into macroscopic phase changes that material goes through. Russo, in [5], identifies four threshold points that define zones of heating, melting, vaporization, mass ionic and electronic plasma creation, and phase explosion. For propulsion purposes, irradiance levels above the vaporization threshold are necessary, because thrust is achieved only by the ejection of the evaporated material.

In this work, the ejection of the material is investigated by shadowgraph visualization, while the amount of the ablated material is deduced from the measurement of the crater volume by means of $3 \mathrm{D}$ surface profilometry.

\section{Experimental methods}

The laser beam used for ablation was an Nd:YAG Continuuum II laser, that emits $7 \mathrm{~ns}$ pulses at 1064 and $532 \mathrm{~nm}$. Both wavelengths were used for the experiments. The energy of the beam is selected by adjusting the delay between flashlamp and Q-switch. The beam was focused by a BK7 converging lens.

Metallic and the plastic targets were of $10 \mathrm{~mm} \times 10$ $\mathrm{mm}$ size, with $1 \mathrm{~mm}$ thickness, and were positioned on a three axis micrometric translational stage, in ambient air. The surfaces of the metallic targets had roughness characterized by the maximum height of the profile (peak to valley height) smaller than $1 \mu \mathrm{m}$, while this value for PVC was about $5 \mu \mathrm{m}$. PVC was of the opaque (black) and hardened type, similar to the type that can be found as a floppy disk cover.

The dynamics of the ejected material in the form of plasma plume was observed and recorded using a standard shadowgraph setup composed of a $3 \mathrm{~ns}$ laser beam as a light source, and a CCD camera as the recording medium. The visualization laser pulse defines the time resolution of the measurement. The plasma which was generated by the impingement the lasers' high energy was very luminous, therefore a series of neutral density filters were used in front of the camera. The camera could take a single image from an experiment, so the sequential development of the plume dynamics was recorded by producing a time delay on the visualization laser through a pulse delay generator. 


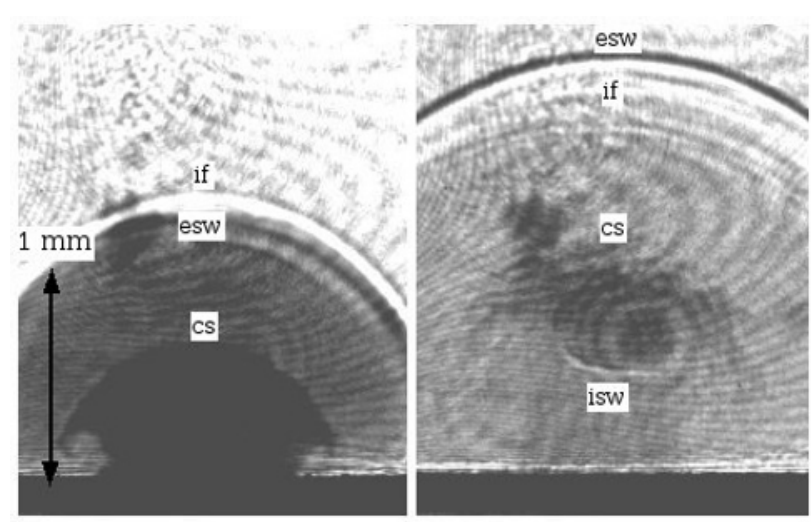

Fig. 1. Shadowgraphs of discontinuities arisen from the laser ablation of copper: if - ionization front, esw - external shock wave, cs - contact surface, isw - internal shock wave.

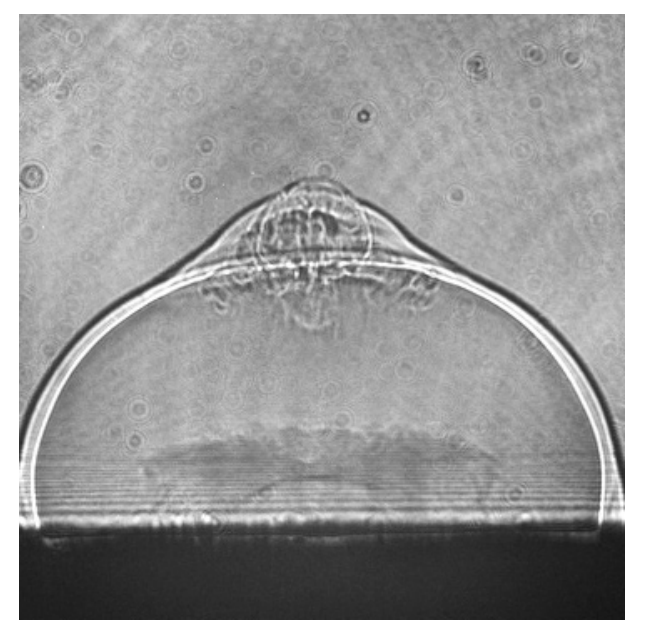

Fig. 2. Shadowgraph of discontinuities arisen from the laser ablation of aluminum and the onset of the laser supported detonation wave. Other discontinuities are also visible. Image was taken 200 ns after the laser pulse.

The repeatability of the phenomena was also tested by taking several images at under the same conditions, with the recorded images being identical within experimental error.

Surface profiling of metals was done with the profilometry based on confocal microscopy technique by using the Nano-focus $\mu$ surf-explorer. The resolution of the instrument is $3.1 \mu \mathrm{m}$ in the plane of the material surface and $2 \mathrm{~nm}$ in along the depth of the material. Since the roughness of the material is of the order of $\mu \mathrm{m}$, the measurement uncertainty in determining the crater depth is influenced by the phenomena, and not by the instrument. Each profile measurement was performed along two orthogonal directions on the plane of the surface.

\section{Results}

A shadowgraph of the laser ablation of copper by a 130 $\mathrm{mJ}$ laser beam (wavelength $532 \mathrm{~nm}$ ) is shown in figure 1. The image on the left is taken $160 \mathrm{~ns}$ after the laser pulse, while that on the right after $300 \mathrm{~ns}$. The laser beam is focused on a spot with diameter of $600 \mu \mathrm{m}$. The images show the removal of the material from the surface and the evolution of generated discontinuities. We distinguish four surfaces of discontinuities: esw - the external shock
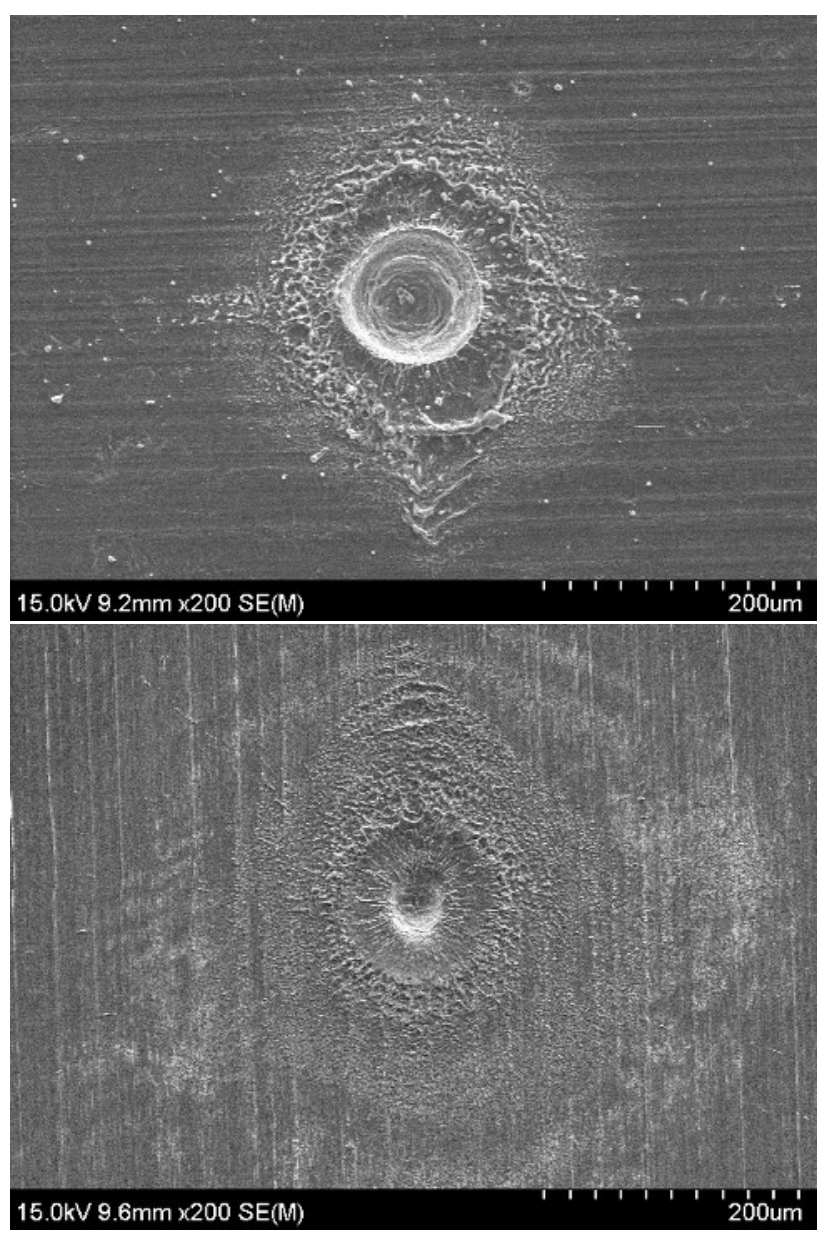

Fig. 3. SEM micrographs of the aluminum (top) and copper (bottom) craters after a single pulse ablation. Laser energy used for ablation of aluminum was $250 \mathrm{~mJ}$, while for copper it was $90 \mathrm{~mJ}$.

wave, which is driven by the ejected material and which propagates outwards from the target and towards the laser; if - the ionization front, which corresponds to the boundary of the plasma luminescence within the region of the shocked ambient gas; cs - the contact surface, which is the boundary of the ejected material; and isw - the internal shock wave, which arises from the continuous ejection of the material from the depth of the target. The internal shock wave initially will move outward from the target, but after encountering relatively cooled ejected material, bounces back toward the target. Numerical simulations by Wen et al., presented in [6], show that the internal shock wave will bounce several times before it disappears, but we captured only once such bounce, because the first ones occur very close to the surface and at very early times when plasma is completely opaque.

The uncertainty in the measurement of time for these experiments depends on the visualization laser jitter. Since the pulse duration is $3 \mathrm{~ns}$, this uncertainty is not longer than $3 \mathrm{~ns}$. The main source of error comes from the uncertainties in determining the position of discontinuities. The images were taken with a $1280 \times 1024$ pixels, and the discontinuity was determined within 4 pixels. Considering the size of the field of view (437 pixels for $1 \mathrm{~mm}$ ), the spatial measurement uncertainty is slightly better than $1 \%$.

A very different behavior of the externally propagating shock wave was observed when the laser beam was 

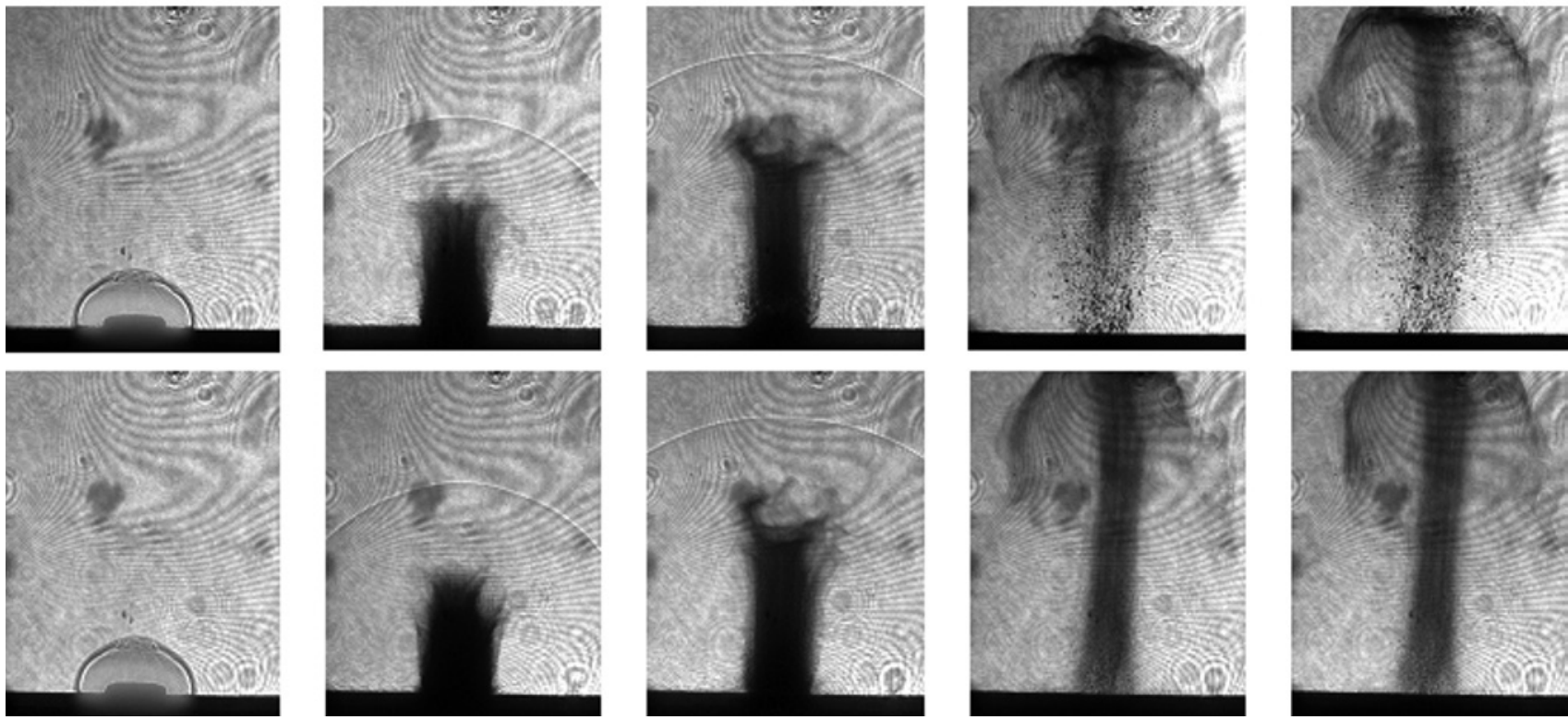

Fig. 4. Expelled material from PVC target after the irradiation by a beam of wavelength $1064 \mathrm{~nm}$ and with $1 \mathrm{~J}$ energy, focued on a spot with diameter of $600 \mu \mathrm{m}$. The images are taken $0.5,2,4,16$ and $18 \mu \mathrm{s}$ after the ablation laser pulse. The dimensions of the view in this image are $7.6 \mathrm{~mm} \times 9.0 \mathrm{~mm}$. Up: a single pulse in a fresh surface; down: successive pulses on the same spot.

focused on a tighter spot, as shown in figure 2. In this case, laser beam (wavelength $1064 \mathrm{~nm}$, energy $1 \mathrm{~J}$ ) is focused onto a $100 \mu \mathrm{m}$ diameter spot, on an aluminum surface. All surfaces of discontinuities are observed, as in the previous case, but now the external shock wave is preceded by a conical shaped wave.

The morphology of the aluminum and copper craters ablated by tight laser focusing, are shown in images of figure 3 , which were produced by scanning electron microscope. The amount of the removed material, though, was calculated by measuring the crater volume, obtained from surface profilometry measurements, multiplied by the density of the material at standard room and pressure conditions.

SEM images show that a deep crater has been created and the melted material has flown outside of the irradiated spot, forming a high rim of order of $10 \mu \mathrm{m}$. In these cases, the rim is quite close to the crater hole, but with the increase of irradiance or with successive pulses, the rim is located further from the hole. In the image we can also see resolidified microdroplets thrown radially. Melt flow is seen only in the vicinity of the rim, which has become a barrier for the melted metal in the crater. Due to quenching, this liquid metal will resolidify in the interior of the crater, forming microdroplets.

On the other hand, as it is expected, ablation of polymers proceeds differently from that of metals. Figure 4 gives two sequences of laser ablation of PVC. The images on top show the ablation in fresh surface, while the images below show the ejected material after successive pulses. Initially, the ablation proceeds similarly for both cases, and we can observe the laser supported detonation wave leading ahead of the rising column of the expelled material. But, around $10 \mu$ s we observe that the ablation of the fresh surface produces granular ejecta, in difference to the previously ablated spot, which produces a pulverized plume. Another observation is that, in comparison to metals, the polymer plume exists for quite a long time (of the order of tens of microseconds, compared to hundreds of nanoseconds for metals), and it is much more massive.

The ablation craters, as shown in figure 5, have great variations in height and depth, which made the measurement of the volume of the craters unreliable, although the ablated zone is clearly observable.

\section{Discussion}

Laser beam impinging on a surface of a metal often is considered to be a heat source that deposits the power density

$$
Q(z, t)=a \mu \exp (-\mu z) I(t),
$$

where $a$ and $\mu$ are the light absorptivity and attenuation coefficient, $I(t)$ is the irradiance as a function of time $t$, and $z$ is the depth inside the metal. But, in the range of irradiances that were used for the presented results $(I=$ $10^{9}-10^{13} \mathrm{GW} \mathrm{cm}{ }^{-2}$ ), optical properties of the material change drastically and light-matter interaction becomes a dynamic process. Therefore, a simple modeling of the ablation processes is not straightforward.

Instead, the phenomena can be approximated by considering the dynamics of the flow of the ablated material. A commonly used method is the application of Sedov theory, [7], which describes the propagation of shock waves generated by an intense explosion at a point. This model can be applied only to the cases shown in figure 1, because there shock wave has a hemispherical shape, testifying for a point-like expansion. The radii of the externally propagating shock waves - as well as other discontinuities, - as a function of time, is given in figure 6 and it can be approximated by the equation

$$
r(t)=C\left(\frac{E_{e s w}}{\rho_{0}}\right)^{1 /(2+\xi)} t^{2 /(2+\xi),}
$$

where $C$ is a constant (usually $C \approx 1$ ), $E_{\text {esw }}$ is the energy content of the shock wave, and $\rho_{0}$ is the density of the surrounding atmosphere. $\xi$ is a parameter that accounts for the 


\section{EPJ Web of Conferences}

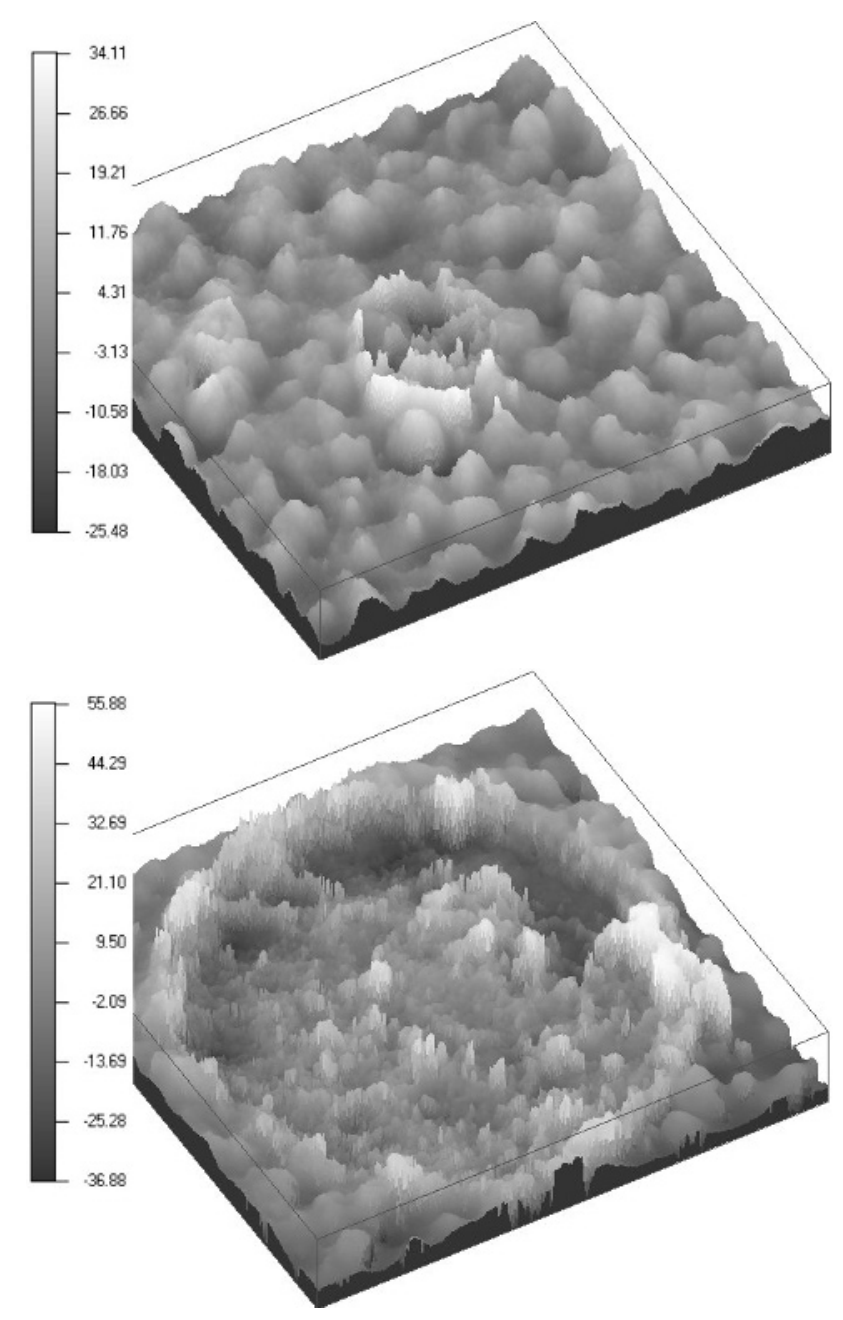

Fig. 5. 3D surface profile measurement of the PVC craters. The length of the measured side is $1.6 \mathrm{~mm}$ and the unit of the magnitude bar height-depth values is $\mu \mathrm{m}$. The image on top corresponds to the ablation by a beam with energy value of $1 \mathrm{~J}$, focused on the surface of the target, while for the image on the bottom the beam with energy of $0.5 \mathrm{~J}$ was focused $10 \mathrm{~mm}$ behind the surface. The material expulsion shown in figure 4 leaves a crater similar to the one in the bottom.

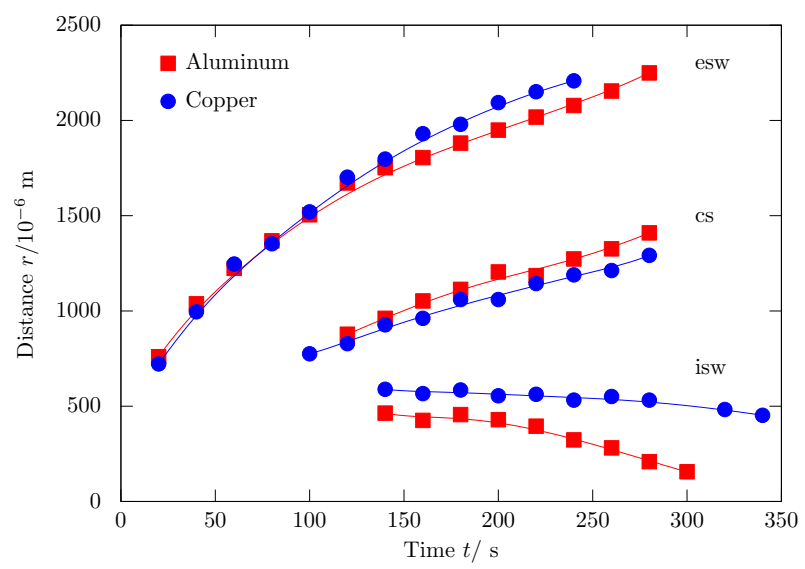

Fig. 6. Distances the discontinuities transverse as a function of time, for aluminum and cooper irradiated by a $360 \mathrm{~mJ}$ laser beam focused onto a spot of $600 \mu \mathrm{m}$ diameter.

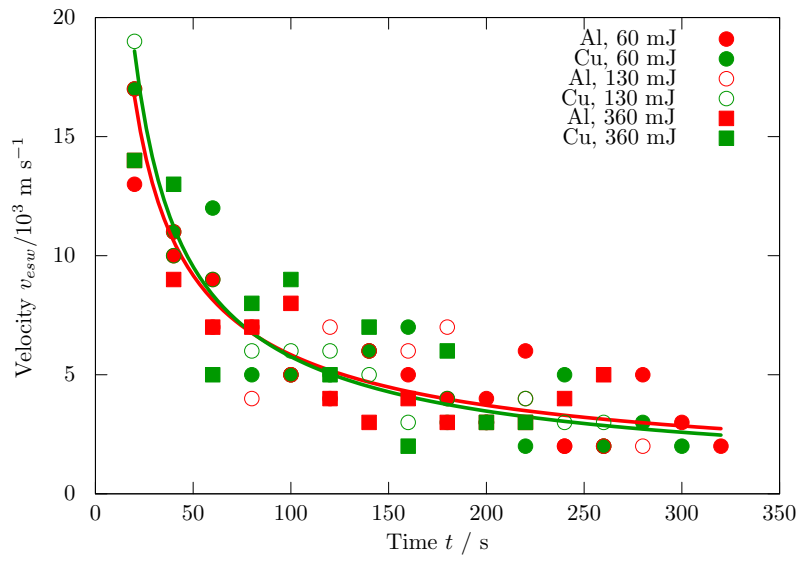

Fig. 7. External shock velocities for aluminum and copper ablated by different energies, as a function of time. Points in the graph present the average velocity calculated from shock positions captured in the images, while the curve is a fit to a power function.

dimensionality of the propagation, and it is equal to 1 for planar propagation, to 2 for spherical, and to 3 for cylindrical propagation of the shock wave.

Considering this theory, our data points for shock position are fitted to a power curve (equation 2), and the values for $n$ change from about 0.55 for lower energies (irradiances $1-50 \times 10^{9} \mathrm{~W} \mathrm{~cm}^{-2}$ ), to 0.4 for higher energies (irradiances $100-5000 \times 10^{9} \mathrm{~W} \mathrm{~cm}^{-2}$ ), regardless of the metal. These values fall between the spherical $(n=0.4)$ and cylindrical $(n=0.5)$ expansions, far from shock propagation with planar symmetry $(n=0.66)$. The reason for the different propagation symmetry of the shock waves lays on the method of achieving high irradiances: if the laser beam is focused tightly, the shock will propagate as a sphere, while if the focal area is relatively large, the shock wave will propagate as a cylinder. In later times, the shock wave becomes spherical in its propagation.

Shock velocity is obtained by deriving equation 2 . Knowing $v_{\text {esw }}$ and applying strong shock relations as a function of shock velocity, we can easily calculate pressure and temperature behind the shock wave. The relation between pressure and shock velocity is given by (see [8])

$$
P_{\text {esw }}=\frac{\rho_{0}}{\gamma+1} v_{\text {esw }}^{2},
$$

where $\gamma$ is specific heat ratio of the surrounding ambient (for air, $\gamma=7 / 5$ ), while the relationship for temperature is

$$
T_{e s w}=T_{0} \frac{\gamma-1}{\gamma+1} \frac{P_{e s w}}{P_{0}},
$$

with $T_{0}$ and $P_{0}$ being room temperature and pressure, respectively. Calculations show that within the first $300 \mathrm{~ns}$, pressure and temperature of the shocked gas just behind the external shock wave decrease from $200 \mathrm{MPa}$ and 100 $000 \mathrm{~K}$ down to $0.5 \mathrm{MPa}$ and $2500 \mathrm{~K}$. The values for the ejected material, though, are an order of magnitude larger, as shown in the study based on laser-induced breakdown spectroscopy [9].

From other discontinuities, the most interesting phenomenon is observed with the motion of ionization front. As it is shown in figure 1, initially the ionization front precedes the shock wave, but later it is overtaken by it. This 
means that at the initial stages of ablation, electrons are the first to leave the surface. Knowing that the refractive index of electrons is lower than one, the effect on the visualization light is opposite to that of the dense gas. After some time (about 100-150 ns), the number of free electrons (or negative ions) becomes negligible, so a standard shadowgraph image is observed (with a dark and bright stripe attached). We attribute this effect to free electrons, rather than negative ions, because it happens at short times and much earlier than the ejection of the plume, so the heavy ions did not have enough time to overcome the inertia.

Contact surface expands almost with a linear velocity, from the first moments when it becomes visible till the moment when it dilutes and its border is not well defined anymore. Internally propagating shock wave initially moves outward as an expanding hemisphere, but then gradually changes into a planar shock, and finally changes its sense of propagation and moves toward the target with speeds of about $2 \times 10^{3} \mathrm{~m} \mathrm{~s}^{-1}$.

For the case with laser supported detonation wave, as shown in figure 2, the detonation-like process starts when the plasma absorbs the incoming laser beam and gains more energy. This has the effect of shielding the surface of target from the incoming laser light, which is confirmed by the measurement of the ablated mass, [10]. Figure 8 shows a comparison of the ablation efficiency with bulk properties of the materials, namely bulk modulus.

The optical properties of a material change with temperature, and once the laser beam impinges on the surface of the material, the temperature of the material changes. This leads to melting and vaporization, and with the increase of temperature, most materials become better light absorbers. But, this also leads to plasma formation, which in turn reflects the light, thus creating a self regulating mechanism. At high irradiances, as presented here, plasma from metals is created during the laser pulse, therefore absorption coefficient can be considered constant. This also explains the (inverse) linear relationship between bulk material properties and the ablation efficiency.

For polymers, such as PVC, the ablation mechanisms are different than those for metals, which have an abundance of free electrons. Most of the initial laser beam that impinges on PVC is spent on breaking the molecular bonds, and the number of ions produced is much smaller, as compared to metals.

On the other direction, part of the absorbed laser energy will initiate a thermal wave inside the target, defining the ablation front, which progresses in the same sense as the laser beam. If the irradiance is higher than a threshold value of the order of $0.1 \mathrm{GW} \mathrm{cm}^{-2}$, then the propagation of the ablation front will cause compression of the solid target, which ultimately generates a shock wave inside the target. At the initial states, the ablation pressure is equal to the asymptotic value of the pressure of the externally propagating shock wave $P_{\text {esw }}$ for $r \rightarrow 0$.

Once generated, the shock wave propagates through the foil as longitudinal compression wave, and its velocity in a thin metal foil can be given by

$$
v_{\ell}=\sqrt{\frac{E(1-v)}{\rho_{m}(1+v)(1-2 v)}},
$$

where $E$ is the Young's modulus of the target material, $\rho_{m}$ is its density, and $v$ is the Poisson's ratio for the material.

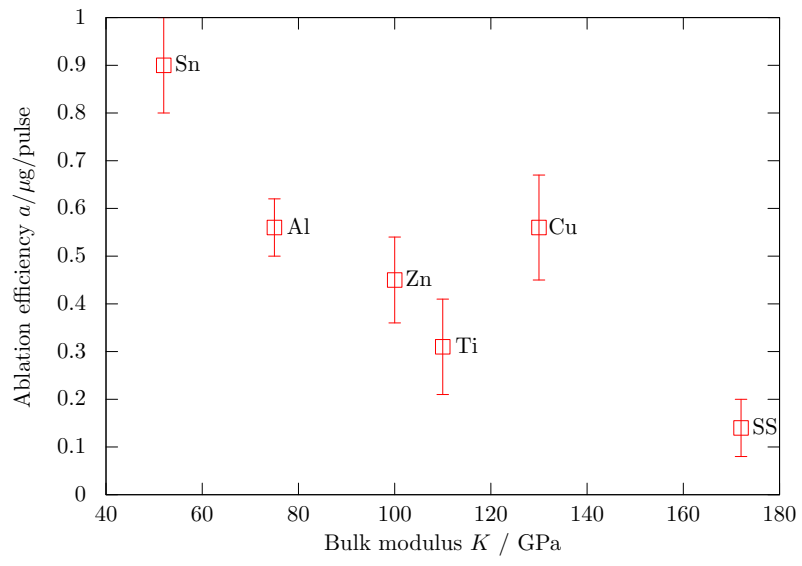

Fig. 8. The ablated efficiency (mass per pulse) as a function of bulk modulus for six metallic materials: aluminum, copper, tin, stainless steel, titanium, and zinc.

When the shock wave reaches the rear end of the target, it emerges from the free surface and the material unloads to atmospheric pressure. This causes a rarefaction wave to travel backward into material with the speed equal to that of sound for the state of the shocked material, and the material itself will move in the direction of the transmitted shock. This motion will result in the propulsion of the target.

An important factor for consideration is the effectiveness of coupling of the wavelength and shock wave. For example, metals are good absorbers of ultraviolet light, and good reflectors of visible and infrared light. But, their breakdown threshold is lower at the ultraviolet light, which results in lower plasma plume pressure peaks. A simple estimate of pressure obtained by laser ablation can be described by the equation

$$
P_{a b l} \approx 40\left(\frac{I}{\lambda}\right)^{2 / 3}
$$

where the ablation pressure $P_{a b l}$ is expressed in $100 \mathrm{GPa}, I$ is expressed in $\mathrm{W} \mathrm{cm}$, and $\lambda$ is the laser light wavelength in $\mathrm{nm}$. A simple estimate shows that pressures of ordero of a few GPa can be easily achieved with laser energies of less than $1 \mathrm{~J}$, if the beam is focused to a spot with diameter comparable to the diffraction limit.

Returning to the expansion of the plume away from the target, it has been shown that confinement of the solid target by a transparent overlay could enhance the recoil pressures several fold (see [4]). This functions because the confining glass prevents plasma from expanding rapidly away from the surface. The result is that the shock wave overpressures are increased, but also the duration of the shock pulse is lengthened. A study from Berthe [11] showed that in the case of irradiation of water confined aluminum foil with irradiances lower than $6 \mathrm{GW} \mathrm{cm} \mathrm{cm}^{2}, 355 \mathrm{~nm}$ light can produce plasma pressures a few GPa higher than $1064 \mathrm{~nm}$ laser beam. But, pressures achieved by this short wavelength will soon saturate, while the infrared beam will continue achieving higher pressures when irradiance is increased

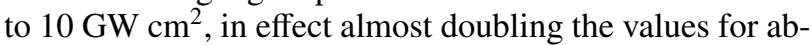
lation pressures. 


\section{Conclusion}

We have presented a collection of experimental results of laser ablation of six metallic materials and PVC. Surfaces of discontinuities of plume expansion for metals were clearly observed and the measurement of their histories facilitated calculation of pressure and temperature behind the leading shock wave, by applying Sedov's blast wave theory. In a parallel study, the values for temperature were confirmed using laser-induced breakdown spectroscopy.

Current study also showed that ablation efficiency depends on bulk properties of the material.

Ablation of polymers showed different features from that of metals, characterized in particular by the massive amount of the ejected material for a duration of two orders of magnitude longer than in the case of any metals. Also, it was noticed that successive ablation produces finer atomization of the plume.

\section{References}

1. D. Bäuerle, Laser Processing and Chemistry (Springer, Berlin, 2011)

2. V. Menezes, K. Takayama, A. Gojani, and S. H. R. Hosseini, Shock Waves 18, 393-400 (2008)

3. T. H. Han, A. B. Gojani, and J. J. Yoh, Appl. Optics 49, 3035-3041 (2010)

4. S. J. Choi, T. H. Han, A. B. Gojani, and J. J. Yoh, Appl. Phys. A 98, 147-151 (2010)

5. R. E. Russo, Appl. Spectro. 49, 14A-28A (1995)

6. S. B. Wen, X. Mao, R. Greif, and R. E. Russo, J. Appl. Phys. 101, 023114 (2007)

7. L. I. Sedov, Similarity and Dimensional Methods in Mechanics (CRC Press, Boca Raton, 1993)

8. Ya. B. Zeldovich, Yu. P. Raizer, Physics of Shock Waves and High-Temperature Hydrodynamic Phenomena (Academic Press, London, 1966)

9. A. B. Gojani, ISRN Spectroscopy 2012, 868561 (2012)

10. A. B. Gojani, j. J. Yoh, and J. H. Yoo, Appl. Surf. Sci. 255, 2777-2781 (2008)

11. L. Berthe, R. Fabbro, P. Perye, and E. Bartnicki, J. Appl. Phys. 85, 7552-7555 (1999) 